Title: Discussion of patient centered care in health organizations

\title{
Authors:
}

First Author: $\quad$ * Please direct questions related to article to this author*

Suzanne Fredericks RN, PhD

Associate Professor

Daphne Cockwell, School of Nursing, Ryerson University

350 Victoria St.

Toronto, ON; M5B 2K3

Office: 416-979-5000 ext. 7978; sfrederi@ ryerson.ca

Fax: 416-979-5332

\section{Second Author:}

Jennifer Lapum RN, PhD

Associate Professor

Daphne Cockwell, School of Nursing, Ryerson University

350 Victoria St.

Toronto, ON; M5B 2K3

Office: 416-979-5000 ext. 6316; jlapum@ryerson.ca

Fax: 416-979-5332

Third Author:

Jasna Schwind RN, PhD

Associate Professor

Daphne Cockwell, School of Nursing, Ryerson University 350 Victoria St. 
Toronto, ON; M5B 2K3

Office: 416-979-5000 ext. 6321; jschwind@ryerson.ca

Fax: 416-979-5332

\section{Fourth Author:}

Heather Beanlands RN, PhD

Director, Master of Nursing Program and Associate Professor

Daphne Cockwell, School of Nursing, Ryerson University

350 Victoria St.

Toronto, ON; M5B 2K3

Office: 416-979-5000 ext. 7972; hbeanlan@ ryerson.ca

Fax: 416-979-5332

Fifth Author:

Daria Romaniuk RN, PhD

Assistant Professor

Daphne Cockwell, School of Nursing, Ryerson University

350 Victoria St.

Toronto, ON; M5B 2K3

Office: 416-979-5000 ext. 6310; romaniuk@ryerson.ca

Fax: 416-979-5332

Sixth Author:

Elizabeth McCay RN, PhD

Chair, Urban Health and Associate Professor

Daphne Cockwell, School of Nursing, Ryerson University 
350 Victoria St.

Toronto, ON; M5B 2K3

Office: 416-979-5000 ext. 6331; bmccay@ ryerson.ca

Fax: 416-979-5332

Conflicts of Interest and Source of Funding

Financial support was received from the Daphne Cockwell School of Nursing Research Cluster Fund.

\section{Acknowledgement}

The authors wish to thank Ms. Kateryna Aksenchuk and Ms. Vanessa Kirunda for their ongoing assistance throughout the drafting of this manuscript. 


\begin{abstract}
The tradition of inherent knowledge and power of health care providers stands in stark contrast to the principles of self-determination and patient participation in patient centered care. At the organizational level, patient centered care is a merging of patient education, self-care, and evidence-based models of practice and consists of 4 broad domains of intervention including communication, partnerships, health promotion, and physical care. As a result of the unexamined discourse of knowledge and power in health care, the possibilities of patient centered care have not been fully achieved. In this article, we employ a critical social theory lens to examine the discursive influence of power upon the integration of patient centered care into health care organizations. We begin with an overview of patient centered care, followed by a discussion of the various ways that it has been introduced into health care organizations. We proceed by deconstructing the inherent power and knowledge of health care providers and shed light on how these long standing traditions have impeded the integration of patient centered care. We conclude with a discussion of viable solutions that can be used to implement patient centered care into health care organizations. This article presents a perspective through which the integration of patient centered care into health organizations can be examined.
\end{abstract}

Keywords: patient centered care, critical social theory, biomedical knowledge, power, health care organization 


\section{Introduction}

Problematic to patient centered care as a viable practice as opposed to a mere philosophy is the long standing tradition of medical power and authority which has shaped health care practice. In using the term "medical" we do not limit this to physicians, but are referring to the inherent power and authority associated with all health care professionals and the health care system as a whole. This social reality of care is socially constructed and has been produced and reproduced over the years. It was generally not common to question the knowledge authority and power of healthcare providers. The advent of patient centered care disrupted the idea of the authoritative healthcare provider and began to deconstruct power relations between patients and providers. Proponents of patient centered care have argued that this approach to care delivery has produced an emancipatory effect through which domination and power in patient-provider relations has been critiqued and examined. ${ }^{1}$

Through the lens of critical social theory, one can see how the power and knowledge of healthcare providers can potentially be oppressive to patients through the imposition of choice and the discursive power of medical values. ${ }^{2}$ Critical social theory assumes that all social systems, including health care, are potentially oppressive. ${ }^{2}$ Considering the patient-provider relationship, this becomes even more evident because of the influences of biomedical knowledge, power, status and issues of access to diagnostics and treatment. In the context of inherent power and knowledge of health care providers, patient values, choices, and free will become influenced and potentially eliminated; this stands in stark contrast to a patient centered care approach in which self-determination and patient participation are underlying assumptions. The tradition of medical power and 
authority is one that is socially constructed and is historically embedded in the functioning of health care organizations and our expectations, actions and interactions as patients and healthcare providers. In order to advance the integration of patient centered care into practice, we first need to examine it in the context of power in health care organizations.

In this article, we employ a theoretical lens of critical social theory and its underpinnings of power to examine the integration of patient centered care into health care organizations. ${ }^{2}$ We present a number of ways in which patient centered care has been introduced into health care organizations. We argue that conscious and critical reflection about the dominant discourse of inherent authority and power of healthcare providers is required before the full possibilities of patient centered care can be actualized. We begin the article with a brief summary of the current state of health care, followed by an overview of patient centered care. Embedded in the discussion of patient centered care, is a presentation of the challenges associated with implementing patient centered care. The final segment of the manuscript attempts to address the tradition of power and authority in healthcare by offering some viable solutions to integrating patient centered care into practice.

\section{Current state of healthcare}

Decreased length of stay, unit and hospital bed closures, and increase healthcare provider-patient ratios have resulted in higher patient turnover, increase hospital readmissions, and the development and/or exacerbation of co-morbid conditions.3 All of which have significantly contributed to an increase in the financial burden on the health care system. ${ }^{3}$ In addition, in the last two decades there has been an increase in advocacy 
groups fighting for patients' rights to have a say in their care. Principles of selfdetermination ${ }^{4}$ and patient participation ${ }^{5}$ in health care have also taken on greater urgency. In response, health care organizations have moved towards revising their existing system of care. ${ }^{3}$ Many organizations have adopted a patient centered approach in which care is based on the collaborative involvement of both the individual and the healthcare provider. In this approach, the patient's desire for information and their involvement in decision making is taken into account and incorporated into the overall plan of care. ${ }^{6-7}$

The transition towards a patient centered approach required health care organizations to ensure the care they would be providing was based on a continuous healing relationship and customized to reflect the patient's needs and values. It was necessary for the patient to be involved in decision making regarding type of care and to have shared knowledge in decision making processes. Additionally, for the outcomes to be effective and in the best interest of the individual, they had to be evidence based. ${ }^{8}$ Patient centered care has evolved over the years and has emerged into different approaches. The following is a description of patient centered care, followed by a summary of the 3 most frequently implemented patient centered approaches that have been adapted by health care organizations.

\section{Patient centered care}

Patient centered care is currently leading health care reform efforts to improve the functioning of health care organizations, specifically in their delivery of quality services, as well as their overall fiscal responsibility. ${ }^{9}$ At the organizational level, patient centered care has been described as a merging of patient education, self-care, and evidence-based 
models of practice. Patient centered care consists of 4 broad domains which include: communication, partnerships, health promotion, and physical care. ${ }^{10}$ This approach to care requires an appreciation of patients' expectations, beliefs, and concerns regarding their illness and/or disease; an understanding of their personal circumstances; the ability to find a common ground on what the problem is, and agreeing on the management; and the knowledge to use the best evidence to inform treatment decisions. ${ }^{10}$

For patient centered care to become a reality, health care organizations have had to invest financially in the development of seminars to train healthcare providers to develop their interpersonal skills so that they are able to work in partnership with patients to establish a collaborative relationship. In doing so, patient centered training seminars have been used to help healthcare providers to develop sincere and professional interest in the experience and concerns of the patients, employ active listening skills, and to ask questions in sensitive ways. ${ }^{11}$ Organizations have also invested in training sessions aimed at enhancing the creation of therapeutic relationships between healthcare providers and patients. These sessions reinforce various aspects of caring that require the healthcare provider to work with patience and sensitivity, accept other's ideas and values, deal with variability, and consider why specific care is planned and the anticipated benefits that will result from decisions made. ${ }^{11}$ The following is an overview of 3 common patient centered approaches adopted by health care organizations.

\section{$\underline{\text { Patient centered interviewing }}$}

Patient centered interviewing involves obtaining data from patients in order to foster the healthcare provider - patient relationship, encourage treatment adherence, and to ensure effectiveness of treatment intervention. ${ }^{9}$ The focus on understanding patients 
and their context is consistent with critical social theory. ${ }^{2}$ The first step of patient centered interviewing involves setting the stage in which the patients are welcomed. During this step the patient's readiness to learn and privacy are assessed by the healthcare provider, barriers to communication are removed, and the patient is put at ease. The second stage of the patient centered interviewing process involves gathering information about the patient's chief complaints and setting an agenda. The healthcare provider usually indicates the time that is available for the interview, obtains a list of the patient's concerns, and establishes an agenda. The provider is enacting a level of power by facilitating and shaping this stage and this may be oppressive to patients as it becomes obvious who is leading the interviewing. The third stage of the patient centered interview consists of the use of open-ended questions to begin the interview session. During this session, the healthcare provider uses attentive listening and pays attention to nonverbal cues. Along the lines of critical social theory, open-ended questioning potentially elicits the patient narrative and engages a dialogical as opposed to a monological encounter. This opens up the possibilities for shared power in the patient-provider encounter. The forth stage of the patient centered interviewing process consists of continuing the interview in which the patient is asked to describe their physical symptoms. The patient's story is focused to include a broader personal/psychosocial context. Emotions that the patient is presenting during the interview are addressed by the healthcare provider by naming, understanding, respecting, and supporting the emotion. Once again, this stage moves beyond the biomedical model, which neglects attention to personal context. ${ }^{2}$ The final stage of the patient centered interview process is the transitioning to provider centered interview in which the discussion from the interview is briefly summarized, the 
accuracy of the interview is checked with the patient, and the provider-centered interview is initiated, in which direct questions related to the chief complaint are asked. ${ }^{9}$

Patient centered interviewing is an approach to patient centered care that is least frequently supported by health care organizations. In the current health care environments, numerous challenges exist to this approach including time constraints, heavy workloads, and competing demands in the clinical areas. It is difficult to engage in this approach when sufficient time is not available to develop a moral and physical proximity with the patient. Further, enhanced skills and training to be able to engage in emotion handling activities are also needed and this is often not addressed in workplace facilitated seminars and workshops. ${ }^{9}$ The quality of the interview is dependent on the skill of the healthcare provider to be authentically present and empathetic in order for patients to feel comfortable in sharing their emotions and discussing sensitive issues. Thus, the completeness of the assessment may vary depending on the abilities of the healthcare provider.

\section{Patient preference}

In attempt to incorporate patient centered care, some health care organizations have started to move towards a patient preference model in which patient's preference for treatment and care is based solely on personal choice that is shaped by the individual's values and attitudes. With regards to critical social theory, eliciting preferences can have an emancipatory effect as patients can potentially feel empowered when being active partners and collaborating in their care. ${ }^{2}$ Patient preference refers to the individual's evaluation of dimensions of health outcomes. ${ }^{12}$ These judgments are expressed as statements or actions. Sidani, Epstein and Miranda ${ }^{13}$ define patient preference as 
reflecting the individual's values and attitudes towards various treatments and interventions. They present three stages associated with patient preference that include: the identification of alternative treatment options that are found effective and relevant, on the basis of best available evidence; the consultation with patients to elicit their preference for the various treatment options; and the inclusion of patients' preference into design and implementation of treatment. Thus, patient preference implies that patients are aware and informed of a variety of treatment options that could be used to manage their presenting problem or improve their health condition. It is up to the patient to decide on which treatment option best suits their needs. As well, information about treatments will influence the patient's values, attitudes, and preferences. Therefore, the emphasis is on the patient to decide on the best possible treatment given a list of choices from which to choose. Patient preference assessment is a collaborative process designed to help patients understand personal values, health care situations, treatment options, and potential outcomes. The assessment is usually conducted by a skilled interviewer.

The integration of a patient preference approach into health care organizations is infrequent, as it requires patients to make decisions under conditions that may be stressful which may in turn cause anxiety and stress. Additionally, patients are required to imagine what their health condition would be like if they selected a certain treatment. This imagination of a future state of health involves abstract thinking, which may not be possible for all patients because of their cognitive level of functioning and/or pre-existing illness conditions. As well, patients with a strong preference for a particular treatment option may refuse or fail to adhere to a treatment if it does not match their preference, which may result in exacerbation of existing illness condition. ${ }^{13}$ Furthermore, not all 
patients may be ready to engage in sharing of decision making. Many patients may prefer

the traditional model of care in which decisions are made by their health care provider. ${ }^{14}$ Moreover, similar to patient centered interviewing, patient preference requires a fair bit of time on the part of the healthcare provider to assess and explain the various treatment options to patients. Finally, the healthcare provider must have the skills to be able to identify appropriate evidence based treatment options before engaging in conversation with the patient and must value patient preference as an essential element of the decision making process.

\section{Self-management support}

Self-management support interventions have also been used to promote patient centered care in health care organizations. There is no single definition for selfmanagement, however it is commonly referred to as the day to day tasks that an individual undertakes to control or reduce the impact of disease on physical health status. It is influenced by education that is delivered with the intent to enhance the individual's skills and confidence in managing their overall health. Self-management support begins during an individual's hospitalization and continues into the post-discharge period. It includes ongoing assessment of knowledge and management of health, goal setting to address health problems, and the provision of problem solving support. ${ }^{15}$ Specific selfmanagement interventions are designed based on five principles that include: the assessment of behaviours and knowledge; the provision of specific information about health behaviours; the collaborative involvement in setting goals between the patient and the healthcare provider; the identification of barriers, strategies, problem-solving techniques; and the creation of a specific plan for follow-up. ${ }^{15}$ Inclusion of self- 
management in the clinical environment requires time, trained healthcare providers, and increased funding to allow for ongoing assessment and follow-up of patients during and after hospitalization.

A self management approach to patient centered care is potentially empowering as patients are collaboratively involved in decision making and asked to be engaged in the day to day practices. Hence, self determination is an important principle as well as patient participation in care. However, similar to a patient preference approach, self management requires a certain level of cognitive functioning in order for patients to engage in collaborative decisions. Potentially, some patients may not be able engage at this level of cognitive thinking and reflection on self care practices in order to make competent decisions. As a result, health care providers need to be skilled at employing a self management approach in ways that it does not oppress patients. For this approach to be effective, the structural resources in terms of both human and educational are critical to its successful implementation.

Implications: Influence of inherent medical power and knowledge on care delivery

The full integration of patient centered care into health care organizations may be impeded by a lack of comfort with wanting to change practice from both the perspective of the patient and the healthcare provider; as well as the inability to be able to partake in the patient centered care related behaviours. Specifically, the power and knowledge of health care provider's, influences the actual and practical potential of patient centered care. We ourselves as health care providers are somewhat oppressed by this tradition. We are caught up in a discursive web that shapes our actions and behaviours based on potent 
historical discourses that lock us in the inert place of comfort. As a result of social, cultural, physiologic, and other factors, many patients do not want to be involved in the decision making aspect of their care. ${ }^{14,16}$ This results in poorer compliance. However, proponents of patient centered care would argue that if patients do not wish to be involved in decision making processes, then respecting their wish is considered to be a form of patient centered care. Similarly, a large number of healthcare providers prefer to engage in the traditional care delivery methods and thus, prefer to provide care that is not reflective of patient centered care principles. Furthermore, many patients and healthcare providers may lack the appropriate knowledge to be able to engage in patient centered care practices, thus settle for the traditional method of care delivery. As well, the acceptance of patient centered care by healthcare providers is limited by practitioners need to achieve their identity. ${ }^{17}$ At present, this identity does not always involve significant sharing of power and responsibility. A shift in perception of identity is required before a stronger patient centered care is seen in practice. ${ }^{17}$

The influence of power and authority on care delivery is also viewed as a motivating factor to engaging patients in complying with treatments. Patients and healthcare providers have different needs and desires related to care which often times may be in conflict. ${ }^{18}$ In such cases, the traditional method of care delivery that revolves around power and authority is implemented by the health care provider to ensure treatments are adhered to. Finally, the engagement in patient centered care can be a financial burden. Healthcare providers end up spending more time with individual patients resulting in a reduced patient load, which may financially impact on specific 
members of healthcare team. Financially, some would argue that it is more feasible to deliver care using traditional delivery methods.

Recommendations: Integration of patient centered care into health care organization

Even though patient centered care is discursively influenced by traditional power and authority, the gradual integration of patient centered care into health care organizations is possible. It begins with a conscious and critical examination and reflection by health care provider of how this dominant discourse limits the full actuality of patient centered care. How does our knowledge and power as health care provider influence patients' decisions? How does it influence whether they are dialogically engaged in the patient-provider relationship? How do health care provider's actions and behaviors lead to oppression or to empowerment?

Significant planning and accommodation is required to advance patient centered care. Change however, is contingent on the values and beliefs of both healthcare providers and patients. Patient centered care has to be a movement that is championed by a group of change leaders including health care providers and patients. In order for patient centered care to become adopted, it requires that health care institutions and health care providers value and recognize the importance of it. Furthermore, various factors need to be taken into account such as the structure of an organization, specifically its infrastructure relating to how decisions are made, staffing practices and relationships, appropriate skill mix, workload patterns, physical facilities, and the potential for innovation and risk-taking. ${ }^{17,19}$ The workload culture relating to the nature of the organization also needs to be accounted for in particular, how things should be done 
within the organization; what is seen as important to focus on and whether or not they relate to the organization's philosophy, value, vision, and mission; and the workplace personality and it many subcultures. Specifically, supportive organizational systems, the sharing of power, and systems that facilitate shared decision making. ${ }^{19}$ Formal and informal communication processes that enable information exchange needs to be considered when integrating patient centered care into health care organizations; as well as the extent to which management, and others are prepared to enact changes in the system related to clinical practice and quality of care issues. The knowledge, skills and attitudes of the target group relate to change, evidence-based practice and clinical nursing excellence need to be also taken into account. Other factors that influence integration of patient centered care into health care organizations include: money, staff education, space and equipment to provide education, clerical support, and retention rates. ${ }^{17}$

As well, even though patient centered interviewing, patient preference, and self management approaches have not been fully actualized in health care organizations, a foundation for implementation has already been established. These approaches can be viewed as a potential model for implementation of patient centered care. Health care organizations should begin to address the identified limitations associated with their specific approach to patient centered care to enhance outcomes.

In order to promote patient centered care, the issue of power inequity needs to be first addressed. This can be achieved, from the perspective of the health care organization, in which specific policies can be designed that describes patient centered care, provides strategies for implementing patient centered care at the mirco-organization level (i.e. individual in-patient units), and which outlines ways in which patient centered 
care can be evaluated. Without specific guidelines for implementation from the health care organization, the transition of patient centered care to the micro-organizational levels will not be successful. In addition, at the micro-organizational level, processes such as workshops and health care provider training sessions, needs to be in place to reinforce the value of patient centered care.

A unique approach to integrating patient centered care into health care organizations at the micro level is the use of coalition building techniques. Coalitions are temporary alliances between individuals to achieve a common goal. ${ }^{20}$ It is a process by which individuals come together to align their strengths to achieve agreed upon outcomes. As a union, individuals are more powerful than on their own. Coalition provides patients with a certain degree of power. It allows the individual to work in unison with their health care provider to identify goals for recovery and maintenance of health, strategies to achieve goals, resources to assist with completion of goals, and appropriate timelines. Coalitions provide patients with a certain degree of power. However, in order for coalitions to be successful, health care providers need to be educated on how to form successful patient coalitions.

Another innovative means in which patient centered care can be integrated at the micro-organizational level is through the use of multi-disciplinary teams to deliver care, with the patient being a valued member of the team. ${ }^{21}$ Processes, such as organizational policies and guidelines, however, need to be established to allow health care providers from different disciplines to come together to work with the patient to identify needs, present strategies to address needs, and techniques for evaluating whether or not health care needs have been met. 
Integration of coalition building techniques and the use of multi-disciplinary teams will have positive implications from a financial and workload perspective.

Financially, if patients are involved in the planning of their care, they are more likely to engage in behaviours to continue to maintain or promote health and/or recovery. Thus, reducing the likelihood for hospital readmissions, which significantly reduces the overall financial burden on the health care system. ${ }^{20,21}$ As well, from a workload perspective, addressing the disequilibrium in patient centered care enhances the likelihood for patients to be receptive to various therapies, thus reducing the health care providers overall workload. ${ }^{20,21}$

\section{Summary}

Power and knowledge can both be facilitators and barriers to the movement of patient centered care. Although we have focused on the impeding influences of power and knowledge in this manuscript, as health care providers we are in a position to speak up, advocate and dialogically engage patients in ways that are empowering. If our inherent knowledge and power remains unexamined, patient centered care will remain a mere philosophy and a practice that never gets the chance to flourish. 


\section{References}

1. Brennan PF Strombom I. Improving health care by understanding patient preferences: The role of computer technology. J Am Med Inform Assoc. 1998;5(3): 257-262.

2. Dickinson J. A critical social theory approach to nursing care of adolescents with diabetes. Issues Compr Pediatr Nurs. 1999;22:143-152.

3. Center for Disease Control Web site. http://www.cdc.gov/chronicdisease/overview/index.htm. Accessed Nov 10, 2010.

4. McCormack B. A conceptual framework of person-centred practice with older people. Int J Nurs Pract. 2003;9(3):202-209.

5. Nambisan P. Nambisan S. Models of consumer value co-creation in health care. Health Care Manage Rev. 2009;34(4):344-354.

6. Hoff T. Managing the negatives of experience in physician teams. Health Care Manage Rev. 2010;35(1):65-76.

7. Gowen CR. Henagan SC. McFadden KL. Knowledge management as a mediator for the efficacy of transformational leadership and quality management initiatives in U. S. health care. Health Care Manage Rev. 2009;34(2):129-140.

8. Institute of Medicine Web site. http://www.iom.edu/Reports/2009/The-HealthcareImperative-Lowering-Costs-and-Improving-Outcomes.aspx. Accessed Nov 24, 2010. 9. Lein C. Wills CE. Using patient-centered interviewing skills to manage complex patient encounters in primary care. Journal of the American Academy of Nurse Practitioners. 2007;19(5):215.

10. Irwin RD. Richardson AND. Patient-focused care: Using the right tools. Chest. 2006;130(1):S73. 
11. Price B. Exploring person-centered care. Nursing Standard. 2006;20(50):49.

12. Rady MY. Verheijde J. Patients best interests standard trumps autonomous healthcare decisions: Paternalism vs. self-determination. Chest. 2010; 38(4):1021-1022.

13. Sidani S. Epstein D. Miranda J. Eliciting Patient Treatment Preferences: A Strategy to Integrate Evidence-Based and Patient-Centered Care. Worldviews on Evid Based Nurs. 2006;3(3):116-123.

14. Sinding C. Hudak P. Wiernikowski J. Aronson J. Miller P. Gould J. Fitzpatrick-Lewis D. "I like to be an informed person but..." negotiating responsibility for treatment decisions in cancer care. Soc Sci Med. 2001;71(6): 1094-1101.

15. McGowan P. Fredericks S. Beatty G. Des Roseier N. Funnell MM. Holubeic I. Johnstone R. Lavoie K. Lyddiatt A. O’Donnell A. O’Halloran K. Schaefer J. Stang C. Stewart-Pyne A. D’Souza E. Strategies to support self-management in chronic conditions: Collaboration with clients. Toronto: Registered Nurses Association of Ontario; 2010.

16.Caress A-L. Luker KA. Ackrill P. Patient-sensitive treatment decision-making? Preferences and perceptions in a sample of renal patients. Nurs Times Res. 1998;3:364373.

17. O'Flynn N. Britten N. Does the achievement of medical identity limit the ability of primary care practitioners to be patient-centred? A qualitative study. Patient Educ Couns. 2006;60(1):49-56.

18. Coulter A. After Bristol: Putting patients at the centre. Br Med Jl. 2002;324:648. 19. McCormack B. McCance TV. Nursing theory and concept development or analysis. $J$ Adv Nurs. 2006;56(5):472-479. 
20. Coalition Building Web site.

http://www.colorado.edu/conflict/peace/problem/coalition.htm. Accessed Jan 25, 2011.

21. The Consequences of Diversity in Multidisciplinary Teams Web site.

http://www.rci.rutgers.edu/ sjacksox/PDF/TheConsequencesofDiversityInMultidisciplin aryWorkTeams.pdf. Accessed Jan 25, 2011.

This manuscript was accepted by Wolters Kluwer Health, Inc, Fredericks, S., Lapum, J., Schwind, J., Beanlands, H., Romaniuk, D., McCay, E. (2012). Discussion of patient centered care in health organizations. Quality Management in Healthcare, 21, 3, 1-8. 